\title{
Resolving The ISM Surrounding GRBs with Afterglow Spectroscopy
}

\author{
J.X. Prochaska* ${ }^{*}$ H.-W. Chen ${ }^{\dagger}$, M. Dessauges-Zavadsky** and J. S. Bloom \\ ${ }^{*}$ Lick Observatory, University of California, Santa Cruz, CA 95064 \\ ${ }^{\dagger}$ Dept. of Astronomy \& Astrophysics and Kavli Institute for Cosmological Physics, 5640 S. Ellis \\ Ave, Chicago, IL, 60637, U.S.A. \\ ** Observatoire de Genève, 51 Ch. des Maillettes, 1290 Sauverny, Switzerland \\ ${ }^{*}$ Department of Astronomy, 601 Campbell Hall, University of California, Berkeley, CA 94720-3411
}

\begin{abstract}
We review current research related to spectroscopy of gamma-ray burst (GRB) afterglows with particular emphasis on the interstellar medium (ISM) of the galaxies hosting these high redshift events. These studies reveal the physical conditions of star-forming galaxies and yield clues to the nature of the GRB progenitor. We offer a pedagogical review of the experimental design and review current results. The majority of sightlines are characterized by large HI column densities, negligible molecular fraction, the ubiquitous detection of UV pumped fine-structure transitions, and metallicities ranging from $1 / 100$ to nearly solar abundance.
\end{abstract}

Keywords: <Enter Keywords here>

PACS: <Replace this text with PACS numbers; choose from this list: http://www. aip. org/pacs/index.html>

Introduction: With the discovery that long-duration $(t>2 \mathrm{~s})$ gamma-ray bursts (GRBs) are extragalactic events [1], it was immediately realized that these phenomenon offer a means of probing gas in the early universe that complements spectroscopic surveys of high $z$ quasars. Identified exclusively with star-forming galaxies [2, 3], GRB events are believed to result from the death of a massive star [4, 5]. The majority emit a bright, power-law afterglow due to the deacceleration of the relativistic jet in the surrounding interstellar or circumstellar medium. The extraordinary luminosity of these events allows for their detection and analysis at very high redshift $(z>10)$, affording the opportunity to study the epoch of reionization when bright quasars were extremely rare [6]. In addition to studies of the IGM, the GRB afterglow spectrum records data on the gas local to the event as well as the interstellar medium surrounding the star-forming region. In turn, analysis yields constraints on the physical conditions within star-forming galaxies in the early universe and also clues to the nature of the GRB phenomenon.

In this proceedings, I offer a pedagogical review of current research using GRB afterglows to probe the universe. Much of the recent advances were enabled by the launch of the Swift satellite, thanks to its rapid localization of GRB afterglows. This proceeding describes the basics of research using GRB afterglow spectroscopy and several of the key results related to the ISM of the host galaxies.

The Experiment: It is expected that GRBs occur within the star-forming regions of high $z$ galaxies. This assertion is supported by the detection of star-forming galaxies coincident with the GRB afterglow and the fact that GRBs tend to occur in the bluest, brightest regions of these galaxies [2, 3]. The sightlines to the GRB afterglow, therefore, 


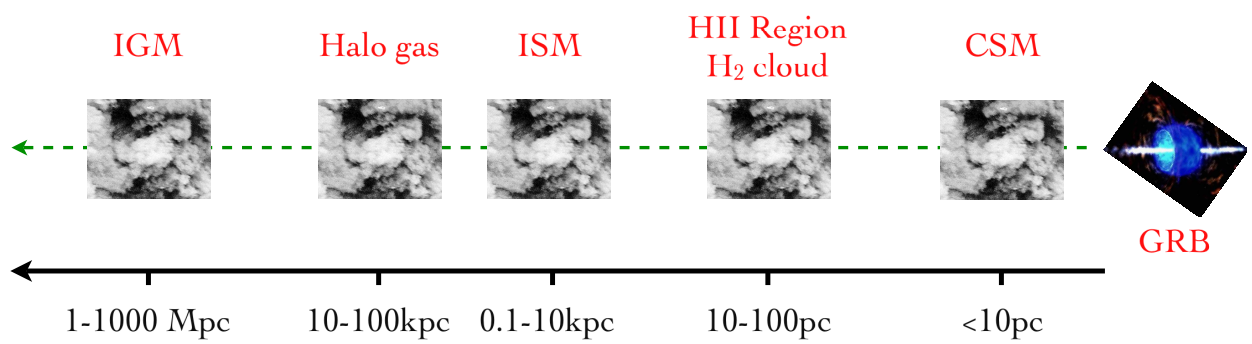

FIGURE 1. Schematic of the various environments intersected by a GRB sightline. Although these phases may be well separated by distance, their absorption features are only distinguished by differences in velocity. Hubble expansion separates the intergalactic medium (IGM), but the various phases within the host galaxy are likely to overlap and one must focus on specific transitions to isolate them.

will travel through (i) the circumstellar medium surrounding the GRB progenitor; (ii) the star-forming region hosting the GRB (e.g. a molecular cloud and/or HII region); (iii) the ambient ISM of the host galaxy; (iv) the baryonic halo of the galaxy; and (v) the intergalactic medium separating Earth from the GRB.

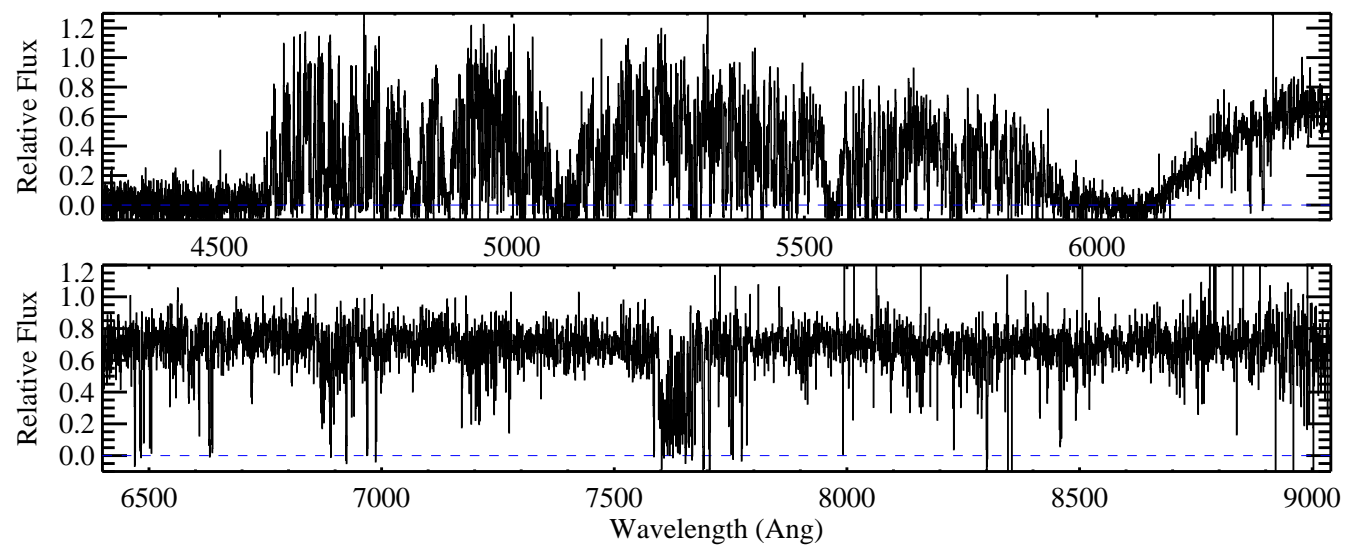

FIGURE 2. MIKE/Magellan echelle spectrum of the bright afterglow of GRB $050730\left(z_{G R B}=\right.$ 3.96855). Imposed on the intrinsic power-law spectrum $\left(f_{V} \propto v^{-1.9}\right)$ are absorption features from the IGM at redshifts $z<z_{G R B}$ and gas associated with the host galaxy of GRB 050730. These include strong Lyman series and Lyman limit absorption by HI gas from the ambient ISM (e.g. Ly $\alpha$ at $\lambda \approx 6100 \AA$ ) and a series of strong metal-line transitions at wavelengths $\lambda>6200 \AA$.

Figure 1 presents a cartoon describing this experiment and the various phases along the sightline. The gas in these various regions will imprint signatures on the observed power-law afterglow spectrum. Ideally, one would separate these spectral features according to the distance of the gas from the GRB to isolate analysis on specific regions (e.g. the circumstellar material). Unfortunately, spectra only distinguish the velocity of gas and one is forced to disentangle these various phases. This is relatively straightforward for the IGM; Hubble expansion gives a lower redshift to all of the gas foreground to the GRB host galaxy. The regions within the host galaxy, however, may share similar velocities and one must identify unique signatures (i.e. specific spectral transitions) to disentangle their imprints on the afterglow spectrum. 
In Figure 2 we present the afterglow spectrum of GRB 050730 obtained with the MIKE echelle spectrometer on the Magellan I telescope [7]. Redward of $6400 \AA$ one observes the intrinsic power-law spectrum of the afterglow due to synchrotron radiation. The absorption features imposed on this power-law spectrum are associated with gas foreground to the afterglow. The thicket of absorption lines at $\lambda<6000 \AA$ are the IGM, the so-called Ly $\alpha$ forest. Similar to the featureless blazars, the IGM reveals the redshift of the GRB, $z_{G R B}$, i.e., the Ly $\alpha$ forest terminates at the redshift of the GRB host galaxy. From the spectrum we estimate $z_{G R B} \approx 6100 / 1215.67-1 \approx 4.0$. The following sections describe a number of scientific results related to studies of spectra like these.

Hydrogen Gas: The strongest features in the spectrum of GRB 050730 (Figure 2) lie at $\lambda<4600 \AA$ and $\lambda \approx 6100 \AA$. These correspond to the Lyman limit and Ly $\alpha$ profiles of the HI gas of the GRB host galaxy. The spectrum easily resolves the Lorentzian damping wings of the Ly $\alpha$ profile which give this spectral feature its name: a damped Ly $\alpha$ (DLA) system. A Voigt profile fit to the line-profile yields a precise measurement of the HI column density, e.g. $N_{\mathrm{HI}}=10^{22.15} \mathrm{~cm}^{-2}$.

Large HI column densities are a common feature in GRB afterglow spectra [11, 8]; GRB sightlines tend to intersect large surface densities of interstellar medium. The median $N_{\mathrm{HI}}$ value is $\approx 10^{21.7} \mathrm{~cm}^{-2}$ and $90 \%$ exceed $10^{20} \mathrm{~cm}^{-2}$ although there is a tail that extends down to $10^{17} \mathrm{~cm}^{-2}$. In contrast to the GRB sightlines, out of the $\approx 1000$ quasar sightlines exhibiting damped Ly $\alpha$ absorption $\left(N_{\mathrm{HI}} \geq 2 \times 10^{20} \mathrm{~cm}^{-2}\right)$, only a few have $N_{\mathrm{HI}}>10^{21.7} \mathrm{~cm}^{-2}$ and none exceed $10^{22} \mathrm{~cm}^{-2}$. This difference emphasizes how GRB sightlines complement quasars observations: GRB sightlines probe the tiny regions with high surface density that are associated with star-forming regions in high $z$ galaxies.

The large HI column densities of GRB sightlines were initially interpreted as the signatures of molecular clouds [12]. The column density distribution, however, includes too many sightlines with $N_{\mathrm{HI}}<10^{21} \mathrm{~cm}^{-2}$ to be uniquely described by this model [8]. Furthermore, as we describe below, the majority of this neutral gas has negligible molecular fraction and is located at $r>100 \mathrm{pc}$ from the afterglow. This distance exceeds all but the very largest molecular clouds in local galaxies. Current expectation, therefore, is that the $N_{\mathrm{HI}}$ values reflect the ambient ISM of the host galaxy [9].

With sufficient blue coverage and resolution, the spectra enable measurements of the surface density of molecular hydrogen $\mathrm{H}_{2}$ along GRB sightlines [11, 14]. Surprisingly, the molecular fractions are very low: $f\left(\mathrm{H}_{2}\right)<10^{-6}$. There are two important implications of these results. First, it demonstrates that the molecular cloud which (presumably) hosted the GRB was photoevaporated, at least along the sightline. The absence of excited $\mathrm{H}_{2}$ states in the spectrum indicates the cloud was destroyed prior to the GRB event. Second, $\mathrm{H}_{2}$ formation is being suppressed throughout the GRB host galaxy. This implies an intense far-UV radiation field, roughly $100 \times$ the Galactic value [14], presumably produced by star-formation throughout the galaxy. This hypothesis should be tested with deep, multi-band images of the GRB host galaxies.

Metal-Line Transitions: In addition to the Lyman series, the GRB spectra generally show metal-line transitions with large equivalent width [16, 17] reflecting the very large HI surface densities typical of the sightlines. Figure 3 presents the diverse set of metal-line transitions observed for two GRB sightlines. These include transitions from 


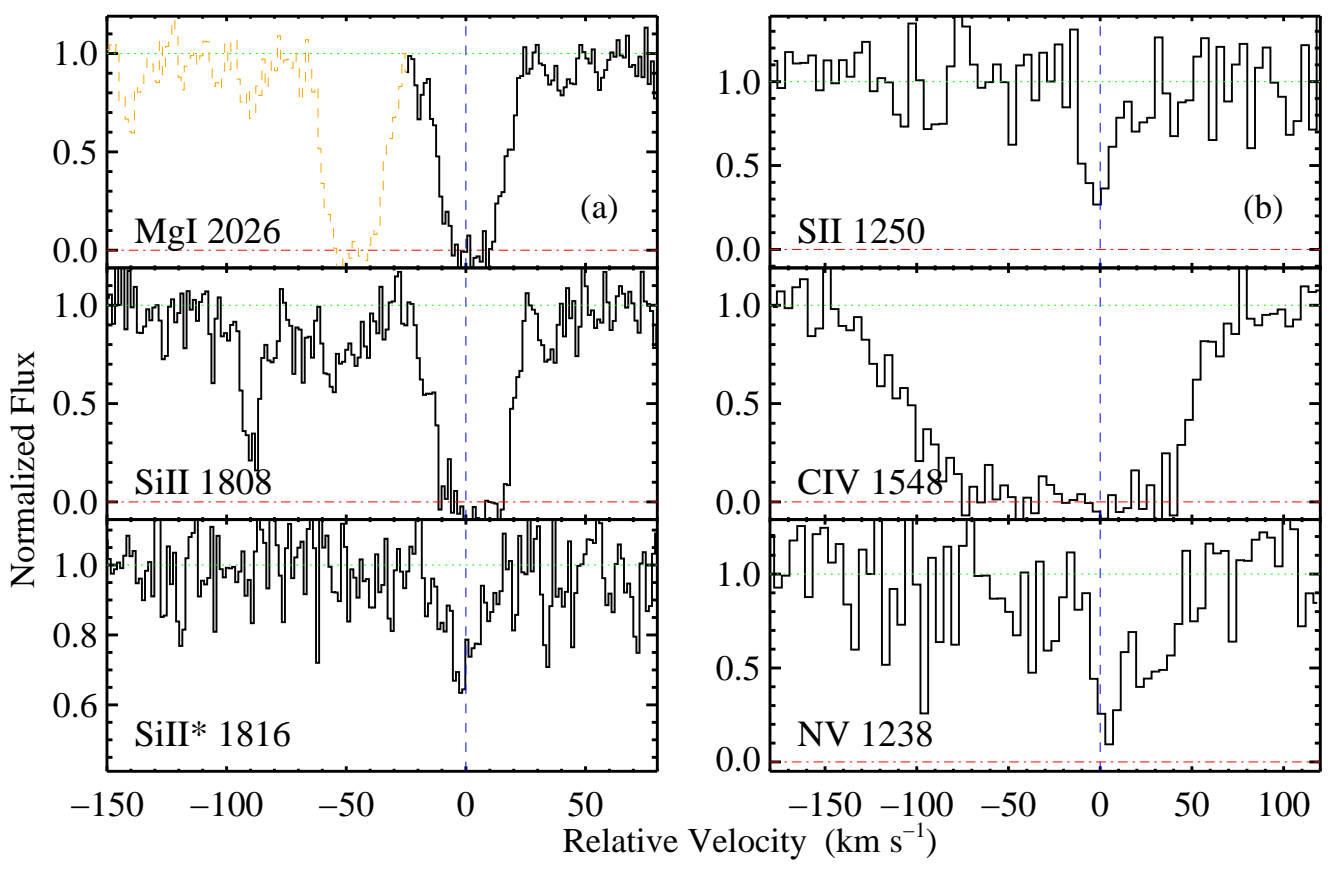

FIGURE 3. Diversity of metal-line transitions identified with the host galaxies of (a) GRB 051111 and (b) GRB 050730 [15, 7]. The MgI and fine-structure (SiII*) transitions constrain the majority of neutral gas to occur within $100 \mathrm{pc}$ to a few kpc from the GRB afterglow. The resonance, low-ion transitions give the metallicity and abundance pattern of the ambient ISM. Finally, the high-ion transitions (CIV, NV) trace the galactic halo and gas near $(r \approx 10 \mathrm{pc})$ the GRB progenitor.

atomic states with ionization potential (IP $<1 \mathrm{Ryd}$; MgI), resonance and fine-structure transitions from low-ion states ( $1 \mathrm{Ryd}<\mathrm{IP}<\approx 2 \mathrm{Ryd}$; FeII, SiII*), resonance transitions from high-ion transitions ( 2 Ryd $<$ IP $<4$ Ryd; SiIV, CIV), and resonance transitions from extremely high-ions (IP> 4 Ryd; NV). This diversity of ionic transitions indicates the sightlines penetrate several phases associated with the host galaxy (Figure 1).

Resolving the Distance of the Gas: The neutral gas observed along GRB sightlines almost universally shows two sets of transitions that allow one to constrain its distance from the GRB afterglow: $\mathrm{MgI}$ and the fine-structure transitions of $\mathrm{Fe}^{+}, \mathrm{Si}^{+}$, and $\mathrm{O}^{0}$. The detection of the former, which coincides in velocity with the low-ion transitions (i.e. bulk of the neutral gas), sets a lower limit of the gas at $\approx 100 \mathrm{pc}$. If this $\mathrm{MgI}$ gas were closer to the afterglow, it would have been ionized prior to the spectral observations [18, 19].

While the detection of $\mathrm{MgI}$ sets a lower limit to the distance of the neutral gas from the GRB afterglow, the ubiquitous detection [16, 11, 7, 22] of fine-structure transitions (e.g. SiII* 1264) places an upper limit to the distance [19]. For a full discussion of the astrophysics of these transitions, see the contribution by P. Vreeswijk. The principle conclusion is that in the GRB environment (i.e. near the extraordinarily bright afterglow) indirect UV pumping dominates the excitation [19]. This has been confirmed by observations of variability in the populations of the fine-structure levels [23, 24]. As the afterglow fades, the UV excitation rate decreases and the majority of excited levels will depopulate. Detailed analysis indicates the gas lies at $100 \mathrm{pc}$ to $2 \mathrm{kpc}$. 
There are a number of implications and applications associated with the UV pumping of fine-structure transitions [19]. First, information related to the density and temperature of the gas has been washed out by the UV pumping. Second, a precise accounting of the chemical abundances of the gas must include these fine-structure levels $(\approx 20 \%$ correction). Third, the detection of fine-structure transitions sets an upper limit on the distance to the gas of a few kpc [19]. Fourth, if one observes strong resonance-line absorption without corresponding fine-structure absorption the gas must be located at distances greater than a few $\mathrm{kpc}$. This has allowed observers to demonstrate that absorption lines previously interpreted as circumstellar material are in fact at much greater distance from the GRB event [25]. It has also been valuable for resolving the origin of low-ion velocity fields [26]. Finally, one concludes that the majority of neutral gas along the GRB sightline is located at $100 \mathrm{pc}$ to a few $\mathrm{kpc}$ from the afterglow, i.e. beyond the immediate star-forming region of the GRB progenitor. While these data do not directly constrain the environment of the GRB progenitor, we recall that much of our knowledge on the progenitor comes from global studies of the host galaxy (e.g. redshift, star-formation history, morphology).

Metallicity: Significant attention has been given to the metallicity of the gas in galaxies hosting GRBs. This has been driven by theoretical expectation that the GRB progenitor should have low metallicity to minimize mass-loss during the lifetime of the star. If the star loses too much mass, it will likely have too little angular momentum to drive the relativistic jet which powers the GRB and its afterglow. Current theory suggests that the GRB progenitors will have metallicities less than 1/10 solar abundance under the assumption of the single-star, collapsar model [27, 28].

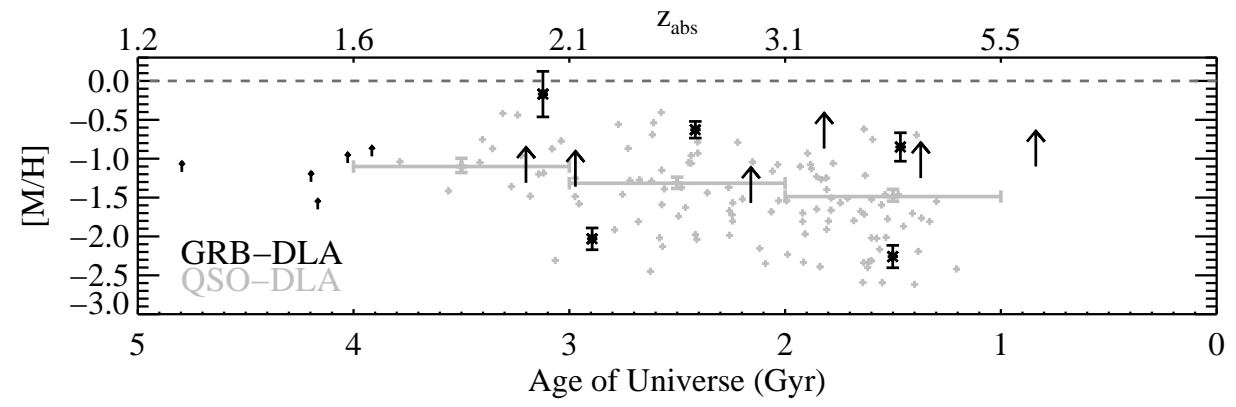

FIGURE 4. Metallicity distribution of the GRB population (dark blue) as a function of redshift. The sample is restricted to $z>2$ where one measures the metallicity from the GRB afterglow spectrum, i.e. a comparison of the metal column density derived from low-ion transitions against the HI column density measured from the Ly $\alpha$ profile [9]. The GRB distribution spans two decades in metallicity and has a median value that likely exceeds $1 / 10$ solar. The green points indicate the metallicities of galaxies (DLAs) intersecting high $z$ quasar sightlines [29]. These also span a wide range of metallicity but have systematically lower abundance than the GRB sample.

At low redshift $\left(z_{G R B}<1\right)$, there is mounting evidence that GRBs preferentially occur in metal-poor galaxies. Imaging of the host galaxies reveal low luminosities and irregular morphologies characteristics of low metallicity galaxies in the local universe [30, 3]. More directly, emission-line diagnostics provide estimates of the metallicity in the starforming regions of these galaxies indicating sub-solar abundances but rarely less than $1 / 10$ solar [31, 32, 35]. 
At high redshift $\left(z_{G R B}>2\right)$, one can measure the metallicity distribution of gas near the GRB using afterglow spectroscopy. With absorption-line techniques one simply counts atoms, avoiding the many uncertainties associated with calibrating emission-line diagnostics. There are, however, a few potential sources of error: ionization corrections, dust depletion, and line-saturation. The first issue is minor because of the very large HI column densities; the majority of gas will be in the low-ion states, i.e. $\mathrm{Si}^{+} / \mathrm{H}^{0} \approx$ $\mathrm{Si} / \mathrm{H}$. Depletion effects are minimized by evaluating the metallicity with non-refractory or mildly refractory elements (e.g. S, O, Zn). The final issue is problematic [37]; with lower resolution or $\mathrm{S} / \mathrm{N}$ data one is generally only able to report a lower limit to the metallicity.

In Figure 4 we present the metallicities of 11 GRBs with $N_{\mathrm{HI}}>10^{20} \mathrm{~cm}^{-2}$ [9]. The sample exhibits a wide distribution of metallicities ranging from 1/100 solar to nearly solar metallicity. Presumably this diversity reflects the diversity of the underlying galaxy population hosting GRBs at these redshifts. Importantly, the distribution is consistent with drawing galaxies randomly according to their current SFR at $z \approx 3$ [39]. The figure also indicates that the GRB metallicity distribution has a median value of at least $1 / 10$ solar; a strict metallicity upper limit is not supported by the empirical observations.

It is also illuminating to compare the GRB metallicity distribution with a control sample. In Figure 4 we present the metallicity values of $\approx 100$ DLAs intervening high $z$ quasars [29]. These galaxies show a similar range of metallicity as the GRB sample yet the distribution is systematically lower [22, 9]. This is likely because QSO-DLA (selected by HI cross-section, not current star-formation) correspond to the outer regions of somewhat fainter galaxies [39].

\section{ACKNOWLEDGMENTS}

J. X. P. is partially supported by NASA/Swift grants NNG06GJ07G and NNX07AE94G and an NSF CAREER grant (AST-0548180).

\section{REFERENCES}

1. M. R. Metzger, S. G. Djorgovski, S. R. Kulkarni, C. C. Steidel, K. L. Adelberger, D. A. Frail, E. Costa, and F. Fronterra, nat 387, 879 (1997).

2. J. S. Bloom, S. R. Kulkarni, and S. G. Djorgovski, aj 123, 1111-1148 (2002).

3. A. S. Fruchter, A. J. Levan, L. Strolger, P. M. Vreeswijk, S. E. Thorsett, D. Bersier, I. Burud, J. M. Castro Cerón, A. J. Castro-Tirado, C. Conselice, T. Dahlen, H. C. Ferguson, J. P. U. Fynbo, P. M. Garnavich, R. A. Gibbons, J. Gorosabel, T. R. Gull, J. Hjorth, S. T. Holland, C. Kouveliotou, Z. Levay, M. Livio, M. R. Metzger, P. E. Nugent, L. Petro, E. Pian, J. E. Rhoads, A. G. Riess, K. C. Sahu, A. Smette, N. R. Tanvir, R. A. M. J. Wijers, and S. E. Woosley, Nature 441, 463-468 (2006), astro-ph/0603537.

4. S. E. Woosley, ApJ 405, 273-277 (1993).

5. S. E. Woosley, and J. S. Bloom, ARAA 44, 507-556 (2006), astro-ph/ 0609142.

6. D. Q. Lamb, and D. E. Reichart, apj 536, 1-18 (2000), URL http://adsabs.harvard.edu/cgi-bin/nph-bib_query?bibcode=2000ApJ...536...1L\&db_key=

7. H.-W. Chen, J. X. Prochaska, J. S. Bloom, and I. B. Thompson, ApJL 634, L25-L28 (2005).

8. P. Jakobsson, J. P. U. Fynbo, C. Ledoux, P. Vreeswijk, D. A. Kann, J. Hjorth, R. S. Priddey, N. R. Tanvir, D. Reichart, J. Gorosabel, S. Klose, D. Watson, J. Sollerman, A. S. Fruchter, A. de Ugarte 
Postigo, K. Wiersema, G. Björnsson, R. Chapman, C. C. Thöne, K. Pedersen, and B. L. Jensen, $A$ \& A 460, L13-L17 (2006), astro-ph/ 0609450.

9. J. X. Prochaska, H.-W. Chen, M. Dessauges-Zavadsky, and J. S. Bloom, ApJ 666, 267-280 (2007), arXiv:astro-ph/0703665

10. J. X. Prochaska, S. Herbert-Fort, and A. M. Wolfe, ApJ 635, 123-142 (2005).

11. P. M. Vreeswijk, S. L. Ellison, C. Ledoux, R. A. M. J. Wijers, J. P. U. Fynbo, P. Møller, A. Henden, J. Hjorth, G. Masi, E. Rol, B. L. Jensen, N. Tanvir, A. Levan, J. M. Castro Cerón, J. Gorosabel, A. J. Castro-Tirado, A. S. Fruchter, C. Kouveliotou, I. Burud, J. Rhoads, N. Masetti, E. Palazzi, E. Pian, H. Pedersen, L. Kaper, A. Gilmore, P. Kilmartin, J. V. Buckle, M. S. Seigar, D. H. Hartmann, K. Lindsay, and E. P. J. van den Heuvel, $A$ \& A 419, 927-940 (2004).

12. D. E. Reichart, and P. A. Price, apj 565, 174-181 (2002), URL http://adsabs.harvard.edu/cgi-bin/nph-bib_query?bibcode=2002ApJ...565..174R\&db_key=

13. N. Y. Gnedin, A. V. Kravtsov, and H.-W. Chen, ArXiv e-prints 707 (2007), 0707.0879.

14. J. Tumlinson, J. X. Prochaska, H.-W. Chen, M. Dessauges-Zavadsky, and J. S. Bloom, ApJ 668, 667-673 (2007), arXiv:astro-ph/0703666

15. J. X. Prochaska, H.-W. Chen, J. S. Bloom, M. Dessauges-Zavadsky, J. M. O’Meara, R. J. Foley, R. Bernstein, S. Burles, A. K. Dupree, E. Falco, and I. B. Thompson, ApJS 168, 231-267 (2007), astro-ph/0611092.

16. A. J. Barth, R. Sari, M. H. Cohen, R. W. Goodrich, P. A. Price, D. W. Fox, J. S. Bloom, A. M. Soderberg, and S. R. Kulkarni, apjl 584, L47-L51 (2003), URL http://adsabs.harvard.edu/cgi-bin/nph-bib_query?bibcode=2003ApJ ...584L..47B\&db_key=

17. S. Savaglio, S. M. Fall, and F. Fiore, apj 585, 638-646 (2003).

18. N. Mirabal, J. P. Halpern, R. Chornock, A. V. Filippenko, D. M. Terndrup, E. Armstrong, J. Kemp, J. R. Thorstensen, M. Tavarez, and C. Espaillat, ApJ 595, 935-949 (2003).

19. J. X. Prochaska, H.-W. Chen, and J. S. Bloom, ApJ 648, 95-110 (2006), a stro-ph/0601057.

20. D. A. Kann, S. Klose, and A. Zeh, ApJ 641, 993-1009 (2006), astro-ph/0512575.

21. R. L. C. Starling, R. A. M. J. Wijers, K. Wiersema, E. Rol, P. A. Curran, C. Kouveliotou, A. J. van der Horst, and M. H. M. Heemskerk, ApJ 661, 787-800 (2007), arXiv: astro-ph/0610899.

22. E. Berger, B. E. Penprase, S. B. Cenko, S. R. Kulkarni, D. B. Fox, C. C. Steidel, and N. A. Reddy, ApJ 642, 979-988 (2006), astro-ph/0511498.

23. M. Dessauges-Zavadsky, H.-W. Chen, J. X. Prochaska, J. S. Bloom, and A. J. Barth, ApJL 648, L89-L92 (2006), astro-ph/ 0606462.

24. P. M. Vreeswijk, C. Ledoux, A. Smette, S. L. Ellison, A. O. Jaunsen, M. I. Andersen, A. S. Fruchter, J. P. U. Fynbo, J. Hjorth, A. Kaufer, P. Møller, P. Petitjean, S. Savaglio, and R. A. M. J. Wijers, $A$ \& A 468, 83-96 (2007), arXiv:astro-ph/0611690

25. H.-W. Chen, J. X. Prochaska, E. Ramirez-Ruiz, J. S. Bloom, M. Dessauges-Zavadsky, and R. J. Foley, ApJ 663, 420-436 (2007), arXiv: astro-ph/0611079.

26. J. X. Prochaska, H.-W. Chen, A. M. Wolfe, M. Dessauges-Zavadsky, and J. S. Bloom, ArXiv Astrophysics e-prints (2007), astro-ph/0703701.

27. S. E. Woosley, and A. Heger, ApJ 637, 914-921 (2006), a stro-ph/0508175.

28. N. Langer, and C. A. Norman, ApJL 638, L63-L66 (2006), astro-ph/0512271.

29. J. X. Prochaska, E. Gawiser, A. M. Wolfe, S. Castro, and S. G. Djorgovski, ApJL 595, L9-L12 (2003).

30. C. J. Conselice, P. M. Vreeswijk, A. S. Fruchter, A. Levan, C. Kouveliotou, J. P. U. Fynbo, J. Gorosabel, N. R. Tanvir, and S. E. Thorsett, ApJ 633, 29-40 (2005), arXiv: astro-ph/0508197.

31. J. X. Prochaska, J. S. Bloom, H.-W. Chen, K. C. Hurley, J. Melbourne, A. Dressler, J. R. Graham, D. J. Osip, and W. D. Vacca, ApJ 611, 200-207 (2004), astro-ph/0402085

32. J. Sollerman, G. Östlin, J. P. U. Fynbo, J. Hjorth, A. Fruchter, and K. Pedersen, New Astronomy 11, 103-115 (2005), astro-ph/0506686

33. M. Modjaz, L. Kewley, R. P. Kirshner, K. Z. Stanek, P. Challis, P. M. Garnavich, J. E. Greene, and J. L. Prieto, ArXiv Astrophysics e-prints (2007), astro-ph/0701246.

34. J. Hjorth, J. Sollerman, P. Møller, J. P. U. Fynbo, S. E. Woosley, C. Kouveliotou, N. R. Tanvir, J. Greiner, M. I. Andersen, A. J. Castro-Tirado, J. M. Castro Cerón, A. S. Fruchter, J. Gorosabel, P. Jakobsson, L. Kaper, S. Klose, N. Masetti, H. Pedersen, K. Pedersen, E. Pian, E. Palazzi, J. E. Rhoads, E. Rol, E. P. J. van den Heuvel, P. M. Vreeswijk, D. Watson, and R. A. M. J. Wijers, nat 423, 847-850 (2003). 
35. N. Mirabal, J. P. Halpern, D. An, J. R. Thorstensen, and D. M. Terndrup, ApJL 643, L99-L102 (2006), astro-ph/0603686.

36. S. M. Viegas, MNRAS 276, 268-272 (1995).

37. J. X. Prochaska, ApJ 650, 272-280 (2006), astro-ph/0606500.

38. J. P. U. Fynbo, R. L. C. Starling, C. Ledoux, K. Wiersema, C. C. Thöne, J. Sollerman, P. Jakobsson, J. Hjorth, D. Watson, P. M. Vreeswijk, P. Møller, E. Rol, J. Gorosabel, J. Näränen, R. A. M. J. Wijers, G. Björnsson, J. M. Castro Cerón, P. Curran, D. H. Hartmann, S. T. Holland, B. L. Jensen, A. J. Levan, M. Limousin, C. Kouveliotou, G. Nelemans, K. Pedersen, R. S. Priddey, and N. R. Tanvir, $A$ \& A 451, L47-L50 (2006), astro-ph/ 0602444 .

39. J. Fynbo, J. X. Prochaska, J. Sollerman, and P. Moller, In prep (2008).

40. B. E. Penprase, E. Berger, D. B. Fox, S. R. Kulkarni, S. Kadish, L. Kerber, E. Ofek, M. Kasliwal, G. Hill, B. Schaefer, and M. Reed, ApJ 646, 358-368 (2006), astro-ph/ 0512340

41. S. Savaglio, New Journal of Physics 8, 195-+ (2006), astro-ph/ 0609489 .

42. M. Dessauges-Zavadsky, J. X. Prochaska, and H.-W. Chen, In prep (2008).

43. G. Garcia-Segura, N. Langer, and M.-M. Mac Low, A \& A 316, 133-146 (1996).

44. B. E. Schaefer, C. L. Gerardy, P. Höflich, A. Panaitescu, R. Quimby, J. Mader, G. J. Hill, P. Kumar, J. C. Wheeler, M. Eracleous, S. Sigurdsson, P. Mészáros, B. Zhang, L. Wang, F. V. Hessman, and V. Petrosian, ApJ 588, 387-399 (2003).

45. R. L. C. Starling, R. A. M. J. Wijers, M. A. Hughes, N. R. Tanvir, P. M. Vreeswijk, E. Rol, and I. Salamanca, MNRAS 360, 305-313 (2005), arXiv: astro-ph/0501120

46. A. J. van Marle, N. Langer, and G. García-Segura, $A \& \&$ A 444, 837-847 (2005).

47. C. C. Thoene, K. Wiersema, C. Ledoux, R. L. C. Starling, J. P. U. Fynbo, P. A. Curran, J. Gorosabel, A. J. van der Horst, L. J. Kewley, A. J. Levan, A. LLorente, E. Rol, N. R. Tanvir, A. de Ugarte Postigo, P. M. Vreeswijk, and R. A. M. J. Wijers, ArXiv e-prints 708 (2007), 0708.3448 ,

48. J. X. Prochaska, M. Dessauges-Zavadsky, H.-W. Chen, and E. Ramirez-Ruiz, apj (2008).

49. C. C. Thöne, J. Greiner, S. Savaglio, and E. Jehin, ApJ 671, 628-636 (2007), arXiv:astro-ph/0611772. 